\title{
Real Time Risk Monitoring in Fine-art with IoT Technology
}

\author{
Mark Phillip Loria, Marco Toja \\ See Your Box \\ 2 Cormont Road \\ London, England \\ SE5 9RA (UK) \\ Email: \{mloria, mtoja\}@ seeyourbox.com
}

\author{
Vincenza Carchiolo, Michele Malgeri \\ Universitá di Catania, \\ Dip. Ingegneria Elettrica Elettronica e Informatica, \\ Viale Andrea Doria 6, \\ 95125 Catania, Italy \\ Email: \{vincenza.carchiolo, michele.malgeri\}@ dieei.unict.it
}

\begin{abstract}
This work presents a bespoke system used to monitor inter-modal logistics within the fine arts industry. A custom IoT architecture provides end-to-end capabilities allowing continuous risk assessment during storage, handling, transport and exhibition. The system overcomes the challenges of implementing adaptive artificial intelligence systems, extra low latency and exceptional power efficiency within a fully integrated IoT architecture. The main contribution of this paper lies in the architecture that has been fully implemented and commercialized to international leading companies.
\end{abstract}

\section{INTRODUCTION}

$\mathbf{E}$ VER since informatics exist, there has been a great interest in the cultural heritage application, many projects aim at improving artwork usability and preservation exploiting some ICT technology and today it is one of the driving forces for both preserving and exploiting Cultural Heritage. Of course, changes in technology and market conditions created and continued to create new and more ambitious challenges [1]. ICT gives rise to a rapid and substantial change in the practice of utilization, supply, and conservation of cultural heritage [2].

Today, virtual reality and IoT are the breaking technologies in cultural heritage and art management. Both technologies play an important role in enhancing the user experience inside real museums, they allow to create complete virtual museums or personalize the surrounding environment. The cultural heritage is changing thanks to the resources offered by ICT technologies. The impact of ICT spans over different application and examples from management to new ways access to arts and culture.

The years to come are going to be interesting and challenging for the use of Internet of Things (IoT) in managing artwork. In this context, some solutions adopted in the supply chain [3] are the starting point for a model to monitor transportation. Indeed, See Your Box ${ }^{\mathrm{TM}}$ Ltd., since its foundation, uses IoT technologies in several applications for arising problems close to artwork management [4], [5].

IoT is one of the ICT technologies that has the largest economic impact on art and culture in many areas such as

This work was funded in part by University of Catania, Dip. Ingegneria Elettrica Elettronica Informatica (DIEEI), under DEDuCE project medicine, energy management and many others. Of course, also in the field of cultural heritage, the IoT is becoming a fundamental technology since it allows the development of several applications covering different objectives. In the last years, two of the main areas in which IoT leads are usability and monitoring.

This work presents a bespoke system with a customized architecture to monitor the transportation of works of art. The system presented in this work has been already implemented and released on the market.

Section II discusses some related works about the use of IoT in the cultural heritage framework. Section III shows the proposed architecture for creating a data collection system for a real time risk monitoring. The main contribution of this paper is discussed in section IV where the architectural solution tailored to inter-modal art logistics processes is presented. Moreover this section discusses the solution proposed by See Your Box ${ }^{\mathrm{TM}}$ Ltd.:

Finally, section V highlights some conclusive remarks with particular attention to the impact of the product on the market.

\section{RELATED WORK}

The most consolidated applications based on the IoT paradigm aim to improve user interactions with museums. There are numerous examples using IoT to craft smart museums [6]. These applications enhance the user experience guaranteeing better interactions with the artworks and their history often realizing smart virtual environments. The main goal of these applications is to renovate the users' interest on the cultural heritage, by enhancing the cultural experiences. Usually, these applications use an indoor location-aware architecture able to provide users with more information and media experience either inside a museum [7] or in open spaces. The most common enabling technologies are (nearly) passive systems such as RFID, Bluetooth, NCS [8][9].

One of the most common examples is the development of a smart guide that helps visitors learn more about artworks in the museum. This application is often enhanced by an artificial intelligence able to recognize speech and/or perform some image processing in order to provide a smart guide. Other interesting examples aim at monitoring the visitors' behaviour in order to 
improve the usability of the museums. For example, Cuomo in [10] uses IoT to collect data about visitor's behaviour and clustering techniques to classify spectators in order to reproduce the visitors dynamics using statistical method.

As previously mentioned, IoT paradigm finds an application in the realization of fine art monitoring systems. Monitoring can be planned in-situ and/or during transportation. Of course, the involved IoT technologies must be tailored to the different use-cases. Therefore the selection of proper IoT technology to monitor the artwork in-situ could be different from the one used to monitor the artwork transportation. The former are IoT indoor technology (e.g. RFID) while the latter create new challenges and interest [11][12]. Usually these applications share the common problem of collecting some sets of values such as, temperature, humidity, vibrations, etc. that can be used to evaluate whether the artwork has been properly managed in-situ or during transportation (e.g. remembering any overtemperature and its length). Moreover, the IoT technologies involved in monitoring during transport must be able to collect also location information. The problems connected to this type of application stem mainly from the sensitive nature of the artworks themselves, because they are fragile against different factors, like vibrations, shocks, temperature, humidity, etc.

A relevant project in monitoring of transportation is PACT-ART [11][13] helps smarten the way transportation processes using external and IoT services to handle tasks depending on their contexts. The system draws reliable predictions about potential misbehaviour and yet-to-come violations.

However the existing solutions often analyze the collected data only at the end of transportation thus they are able only to signal eventual anomalies. Indeed, they cannot perform any action that can try minimize the impact of the anomaly so reducing eventual damages. We believe that the capability to avoid or reduce the damage is the main matter both in economic terms and in cultural and historical terms, than the solution proposed in this paper.

\section{Motivation}

In this section we discuss the proposed architecture for creating a data collection system for a real time risk monitoring solution tailored to inter-modal art logistics processes. Monitoring risk in a inter-modal logistics processes represents a number of challenges for the ICT solutions involved. Across the process multiple stakeholders are concerned about very different KPIs while goods are handled in different ways and challenges can be of different nature. Furthermore, as it may sound, the actual shared goods across the process are not the paintings but risk. Risk for the collector is the health of the paint on an ancient unique painting, risk for the logistics company is tied to the handling of an insulated shock absorbing crates, for a museum it's temperature control inside a safe and so on.

The further challenge for the data collecting solution is that risk is not necessarily (only) directly related to plain data. A very good example is described in [11]. The integrity of an art piece depends on temperature. Supposing the painting is transported in an insulated case, a measured value approaching critical levels might be absolutely fine if the external temperature exhibits an opposite trend. On the contrary, small variations measured inside the crate could be an important indicator of either a damaged box or a dangerous condition. In other words the temperature level measured close to the painting is not sufficient to determine the level of risk without knowing the temperature outside the box, the time of year and day, the latitude, weather forecast and how long the painting is expected to stay in that condition. Data without specific context in an inter-modal monitoring provides data that most of the time are either incomplete or irrelevant. In literature it's possible to find many references on work performed in the field of context aware data analytics systems. These make it possible to identify the different phases of an inter-modal logistic process and adapt the analytics and business intelligence accordingly. As an example, taking into consideration data collected from an accelerometer sensor, the same data set could have two completely different meanings if collected during two different steps of the logistics process. In figure 1 samples collected at $100 \mathrm{~Hz}$ from a high precision MEMS sensor during transport reveal a perfectly safe condition since the resulting $\mathrm{G}$ force is always below the maximum allowed threshold of $3 \mathrm{G}$ for fine art. However, when the same data is sampled in a stationary context 2 the system is able to detect a potential threat since the art piece is supposed to be absolutely still during an exhibition.

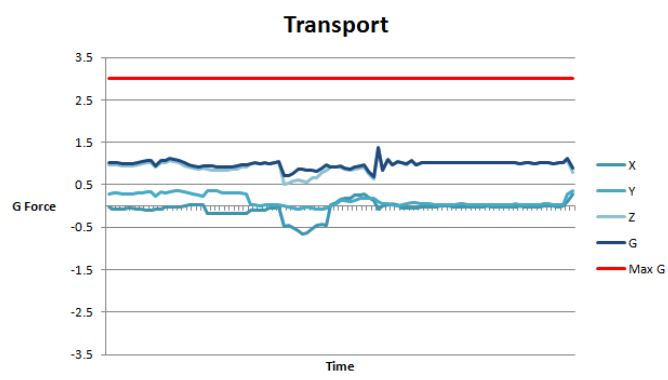

Fig. 1. Accelerometer profile during transport context

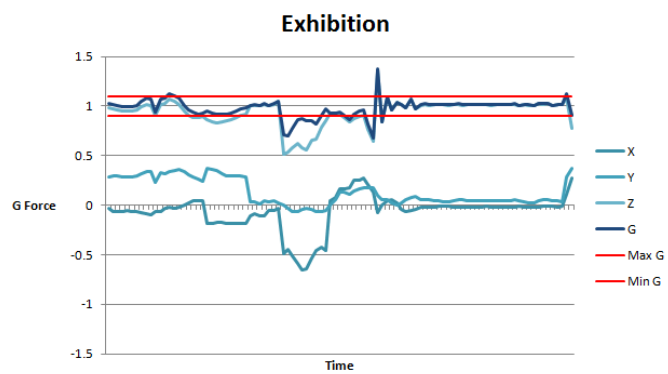

Fig. 2. Accelerometer profile during exhibition context

It's also possible to find in literature references on contextaware monitoring solutions targeted to the fine art logistics 
industry and the focus on this specific field is particularly relevant due to:

- The economic and cultural value of the moved goods

- The goods are typically unique and not replaceable

- The high insurance premiums for theft and mishandling

- The phases of the logistics process are standard and well planned

However, while tackling sharply the matter from a data analytics view point, many solutions overlook how to source data of moving art pieces. In this paper we want to shift the focus from data analytics and information extraction to the data collection phase. Data analytics is surely a great tool for extracting a truthful risk model that can lead to better understanding of the underlying patterns. On the other hand, due to the value and uniqueness of fine art pieces stakeholders are demanding early or real time access to a measure of risk profile for the art pieces while they are moved across the globe on an inter-modal process. This step represents one of the most challenging components of a real time monitoring system for fine art logistics, especially in the case of temporary exhibitions. This implies that any data collection system must be able to record and transmit data globally without the need of a dedicated telecommunication infrastructure. The system must be battery powered, wireless and its dimensions must be such to allow a pervasive monitoring of the art piece. These points lead to a real time monitoring system that must be adaptive from its core by adapting not only the analytics but also how data is collected. The system must also be able to distinguish among different masterpiece classes. Paintings differ in material and era, and are not to be treated as sculptures or jewelry. Inter-modal risk monitoring requires therefore an embedded AI system capable of adapting both to a variety of goods and a variety of logistics scenarios. If not, such lack would force to create so many different technologies that would result in an unsustainable business model.

Following the previous example, the effects of lowering the accelerometer sampling rate to reduce the amount of data transmission in order to contain battery drain and telecommunication costs, could have potentially dangerous effects. In figure 4 we show how lowering the sampling rate effects the resulting data acquisition and subsequent analysis.

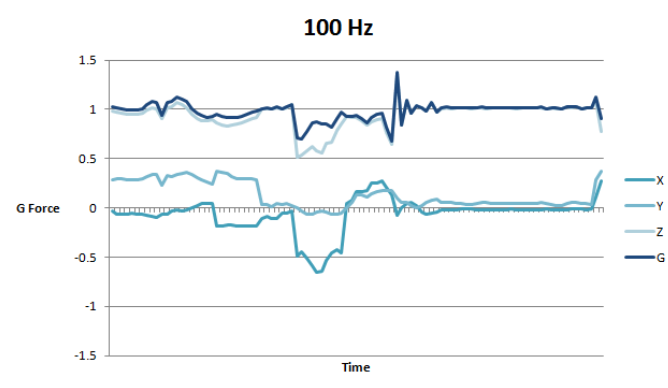

Fig. 3. Accelerometer profile sampled at $100 \mathrm{~Hz}$

During transport on road low intensity high frequency vibrations caused by rough tarmac or cobblestone can have

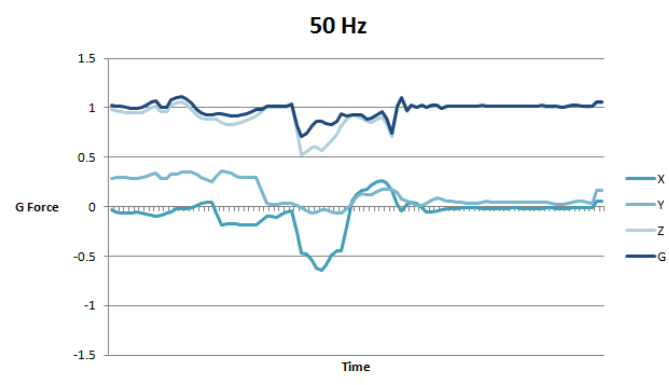

Fig. 4. Accelerometer profile sampled at $50 \mathrm{~Hz}$

devastating effects on moving art pieces. When a design setting such as accelerometer sampling rate is fixed to a low value to contain battery drain during the months of stationary exhibition it could totally miss capturing potentially dangerous situations that could occur during the few hours of road transport. On the other hand configuring the system for a worst case scenario would have negative effects on battery drain, transmission costs and memory usage during periods of signal loss when data is buffered. The challenges encountered have already been a topic of research and we identified two macro categories of approaches:

- Centralized monitoring - Data is collected by simple sensors that have no logic applied. This approach is typical of wireless sensor networks. A central node with high computational power processes incoming data to detect a event

- Distributed monitoring- This approach is typical of edge computing or smart sensors. These have enough processing power and a sufficient vision of the environmental conditions to detect events.

Both approaches have their limitations when confronted with the challenges of fine arts. In a centralized approach customization of sensors is done at design time. Elements such as sampling frequency, resolution and power management are set for the whole process leaving very little room for optimization. Sensors are very cheap due to the low complexity but require continuous stream of data to the centralized node. By leveraging edge computing, distributed systems overcome the issue of data transmissions at the cost of complex and more expensive sensors. These however are often lacking a complete overview of the process since context awareness is achieved by the interaction with other sensors and require high computational power in devices that need to be almost disposable and therefore are cost sensitive. These considerations lead us to the architecture presented in this paper, that is a fusion of both approaches. A high performance cloud based central node continuously segments the risk model into sub-models for each one of the inter-modal logistics phases translating them into simple configuration parameters for the sensing nodes. When the system detects the occurrence of an event or change in context the parameters are uploaded to the distributed nodes. The rationale behind the architecture is quite 
simple, since only a node with full vision over the process can determine what risk sub model fits the process at the give time. Understanding these restrains and specific legal requirements leads to build a dedicated hardware and software architecture where the key elements are the speed, size and content of the package you transmit.

\section{ARCHITECTURE}

The system is composed by two main elements that are the smart sensing devices and a centralized cloud based infrastructure with a high performance rules engine that processes incoming data. Due to the volume of data the whole cloud platform is built upon a totally virtualized environment to allow instantaneous scalability. A web-based management dashboard offers real time access to the risk monitoring platform while REST APIs enable full M2M integration with the system. The architecture of the cloud platform has been described in detail in previous work. In the rest of this chapter we will discuss in detail the architecture of the smart sensing device.

\section{A. Smart sensing devices}

The sensor nodes are based on a proprietary hardware platform. One of the key ideas of IoT for industrial applications is the possibility of digitalizing a process by capturing data points directly on goods. A concept built on the experience of the flight simulators for military applications that is seeing new usages in the industrial world, is the concept of digital twin. Data collected on the real object feeds a computerized model that is used for predictive analysis and simulation. To achieve the necessary level of confidence it's mandatory to have sensors that can be placed as close as possible to the real object and capture key parameters. In the case of fine art paintings this means placing the smart sensing devices directly on the picture frame as close as possible to the canvas and not on the building or vehicle. In figure 5 a smart sensing device is placed directly on a picture frame during the packaging phase and will be removed only after the end of the whole logistics process six months later.

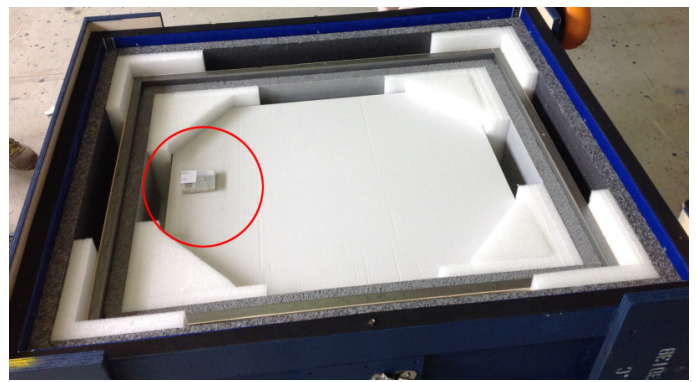

Fig. 5. A SYB device placed directly on a painting frame before placing inside transport box

This creates a number of challenges, specially for end-toend monitoring. From a hardware and mechanical view point the smart sensing devices will have strict constraints on size, weight and type of batteries. From a usage process view point the device must be completely autonomous after power on and require no human intervention until completion of monitoring. From a software view point the smart sensing device must be able to collect and process data as specified by the risk sub-model, guarantee time synchronization of collected data, manage power and data transmission efficiently and lastly ensure a high level of data protection for transmitted and stored data. Figure 6 summarizes the functional blocks of the smart sensing device and in the rest of this paragraph we will go into detail for the most important ones.

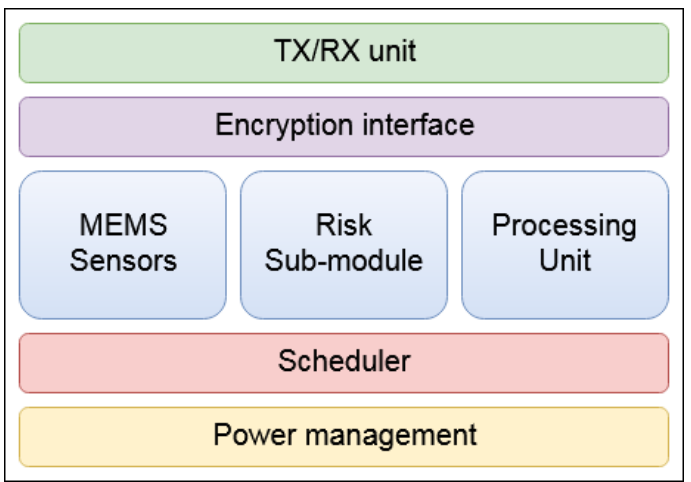

Fig. 6. Functional blocks of the smart sensing device

1) Risk sub-model parameter set: In the hybrid architecture we are proposing elements of the risk model are shared with the sensing nodes. Transferring a whole risk model, that can easily be as large as $2 \mathrm{MB}$, to the edge nodes would raise the minimum requirements in terms of memory, processing power and transmission costs. On the other hand these devices must be as simple and low cost as possible since in many situations they might need to be considered as disposable. The underlying idea is to first break up the risk model into sub-models, one for each phase of the process, and subsequently translate them into a small set of incremental parameter changes for the smart sensing device. As an example, accelerometer impact profiles and vibrations measured over more than 100.000 working hours of monitoring service were divided into risk classes. Using data mining techniques for each class we were able to extract key parameters for the processing unit that detects potential impacts such as maximum $\mathrm{G}$ force during impact, minimum sampling frequency, low-pass and high pass filters, vibration waveform and so on. To further optimize the transfer of these parameters we restricted them to a discrete amount of reasonable values and structured them inside configuration registers. To put this into perspective with a practical example, when the transport of a painting is completed and it is placed into the museum the change of parameters is performed with the transmission of only 8 bits of data. This is sufficient to instruct the smart sensing device to change the type of accelerometer event to detect and adjust the thresholds accordingly. It is important to underline that this simplification of the risk sub model affects only the smart sensing nodes and data incoming to the cloud platform is processed using the full risk sub-model. Of course when using a discrete amount of parameters it is only possible to customize the functionalities 
at run time according to what has been defined at design time. This however is not a limitation since the responsibility of the smart sensing device is not to determine if the actual event has happened (calculating therefore risk) but collecting relevant data for the centralized processing node. The collection of false positives actually is beneficial for the system to feed the data mining algorithm with more balanced data. The risk submodel parameter set is not only in charge of determine how data is collected but also how data is transmitted by the smart sensing device. On this matter there are at least three crucial elements that it must manage. The first one is instructing the smart sensing device on contexts where data transmissions are not allowed, the second one is optimizing means of data transmission for costs and the third one is power consumption optimization. During end-to-end monitoring for temporary exhibitions it is not uncommon to have to perform a logistic step over air freight. Precise regulations (quote) specify how and when portable electronic devices are allowed to be active and transmit. In the case of fully autonomous devices such as the ones we are describing in this work, this creates a strong challenge to guarantee the compliance with existing regulations while the system relies totally on AI. To achieve a high level of reliability it was necessary to create a three level failsafe aircraft detection algorithm that uses data from three different sensors. All related algorithms have been coded in negative logic so in the event of failure on one sensor the smart sensing device will enter a protection mode and disable transmission not to risk transmitting while on an aircraft. Art pieces moving around the world and actively transmitting data requires careful attention on optimizing what channels to use to transmit data not to incur into heavy roaming chargers. The risk sub model contains all necessary parameters to regulate what channels to use, for instance GSM rather than $3 \mathrm{G}$ data, to optimize not only cost but also power consumption.

2) Scheduler: In the previous section we went through an example regarding the customization of the accelerometer. The smart sensing device is equipped with 7 physical sensors and is able to interpolate them to create virtual sensors. An example is crash detection that is achieved with an interpolation of data from accelerometer and microphone. For each sensor the smart sensing device can set thresholds, sampling rate and pre processing data algorithms to execute. The internal scheduler acts as the heart of the smart sensing device, regulating precisely how data is sampled from sensors and generates all necessary tasks that in turn are executed. Lastly, and most importantly, it is in charge of keeping all data collected in sync with the time reference of the centralized node. The scheduler and hardware architecture are heavily coupled. During system design we identified two macro approaches that were either adopting a high performance micro controller with low cost simple sensors or a simpler processing unit and more complex MEMS sensor that could be programmed. The former was quickly discarded since it would have required almost for sure a real time OS and the designing of a scheduler with a high frequency internal rate to poll all sensor at an adequate rate. The latter, on the other hand allowed us to design a simpler scheduler, run with a slower internal clock and ultimately lower power consumption. In the final implemented design all interactions with sensors are based on an asynchronous approach leveraging internal threshold based interrupts. This allows us to keep the main scheduling loop for task management to a very low frequency. When collecting data used to generate a risk profile it is absolutely crucial to guarantee perfect time synchronization and to know exactly when an event has actually happened. Smart sensing devices, or any remote node in general, will be subject to two challenges related to time synchronization of data. The first one is managing offset synchronization while the second one is managing imprecision on the oscillator of the devices itself. This is particularly relevant when designing an ultra low cost device that can collect data in hostile conditions since temperature can affect the precision of the oscillator. Common solutions, such as synchronization with external references like GSM networks or GPS satellites were not viable. Currently there is no international agreement on time synchronization between GSM providers worldwide and the availability of GPS coverage can be a serious issue for indoor monitoring such as museums and safes. To further challenge the system, to contain power consumption active components (and therefore clocks) are off or in an ultra low power mode during most of the time. The adopted strategy was to use two internal clocks (readily available in the chosen micro controller) to self calibrate the real time clock used by the scheduler. With this simple solution we were able to increase the accuracy of a factor of 10 while not introducing extra hardware. A second later of synchronization to manage potential drift issues is introduced on the central processing node. This layer uses a tick based approach were incoming time references from the smart sensing devices are compared with NTP servers and samples are repositioned in time using a linear projection. While introducing an element of complexity due to a dedicated time synchronization algorithm this approach allowed the system to achieve a higher level of accuracy in harsh conditions over alternative solutions such as one-time synchronization coupled with a CMOS backup battery. As an added benefit, it also allows to contain cost and space on the PCB.

3) Power management: The smart sensing device can be used for monitoring long term end-to-end exhibitions that can last up to six months. To allow the device to be placed directly onto the picture frame weight and dimensions of the batteries must be contained to a minimum. Therefore it's necessary to enforce strict power management techniques to minimize consumption. The peculiarity of end-to-end intermodal logistics transports is that data can be collected and transmitted in very different ways. As an example, to ensure that paintings are transported onto the safest and fastest route it is crucial that location updates must happen every 10 minutes to allow the centralized node to detect potential problems like the presence of exceptional traffic and therefore allow a rerouting solution in time. During an exhibition on the other side daily data updates are sufficient. These are two examples of how it's possible to leverage the context of two 
different steps within the logistics process to optimize power consumption. However, transmission rates are not the only area of possible intervention. Other examples are the need of having an RF interface always on to receive asynchronous requests from the central node. These are crucial during transport to allow the central node to ping the location of the smart sensing device at any time. It's not always possible or optimal to set these parameters within the risk sub-model and statically impose them to the scheduler. The reason is that many of these decisions require a large amount of environmental parameters and transmitting them to the centralized node would defeat the objective of reducing the number of transmissions and quantity of data. The key idea we are using is to insert within the risk sub model parameters that can define if a specific power saving strategy is allowed or not and let AI algorithms on the smart sensing device make the final decisions. The challenge of this approach however is that while it's possible to find many algorithms and tools with high levels of abstraction that are easy to implement and run on a server, scaling them down to an ultra low power micro controller with limited quantity of memory and resources requires custom solutions and proprietary algorithms.

4) Data processing: One of the benefits of not creating a purely telemetric system is that data can be processed on the edge node. This also allows us to heavily reduce the amount of data that is sent from the device to the centralized node during a standard end-to-end fine art logistic process that could easily last over six months. A telemetric system that monitors acceleration at $50 \mathrm{~Hz}$ would need to transmit, supposing 16 bit resolution for each axis, over $4.5 \mathrm{~Gb}$ of raw data streamed to the centralized node. From a cost perspective this absolutely not acceptable since part of inter-modal logistics are international and transmissions would incur into massive roaming charges. Even buffering chunks of 1 minute would always mean facing over 250.000 data transmissions. The battery would not last more than a day. On the other hand a system with edge computing allows us to implement a much more efficient strategy. If we are monitoring impacts we can transfer the maximum impact and type of event to detect within the risk sub-model using 16 bits and each acceleration peak would only require 6 bytes of data to transmit. Even if the risk sub-model on the server would require a full minute of data recording to analyze thoroughly the impact we would have reduced the amount of data to $18 \mathrm{~Kb}$ for each potential impact event (16bits per axis sampled at $50 \mathrm{~Hz}$ for a minute). The basic strategy is to use the risk sub-model parameters to reduce the amount of data by sending data with a higher content of information and less noise.

5) Security: The collection of data related to things is a sensitive topic as it raises many concerns regarding security. While security protocols and policies are not a new topic and we can rely on well established best practices for IP based solutions, when scaling them down to low power IoT devices they either result cumbersome or simply not relevant [14]. Designing an effective solution for IoT is still an open challenge [15]. In the fine art logistics industry the major challenge is protecting data from malicious activities related to theft. It was reported that it took only 20 seconds to steal fine art jewellery from an Italian museum causing a total loss of several million [16]. Such efficiency is generally achievable only after a careful study of guard habits and security measures of a museum. Today smart sensing devices are used during exhibitions to monitor the passage of guards and ensure that the painting is safe. Should this data become available to a thief it would put the safety of the painting at great risk. To protect the system prior to a potential theft it is essential that the device and transmission protocols offer a form of protection against eavesdropping. All data exchanged between the smart sensing device and the centralized node is sent over encrypted channels that use a proprietary encryption algorithm where a symmetric encryption key is transferred over a dedicated channel. In the unfortunate event of theft the system must be able to face at least two other types of attacks. These are typically replay attacks and man-in-themiddle attacks. These types of attacks, if successful, could allow a thief to fool the system by making it believe that the painting is still located in the same position or even worse alter data altogether. In both cases risk is heavily mitigated by the use of encryption, nonces and hardware anti tampering mechanisms on the device itself.

\section{B. Cloud platform}

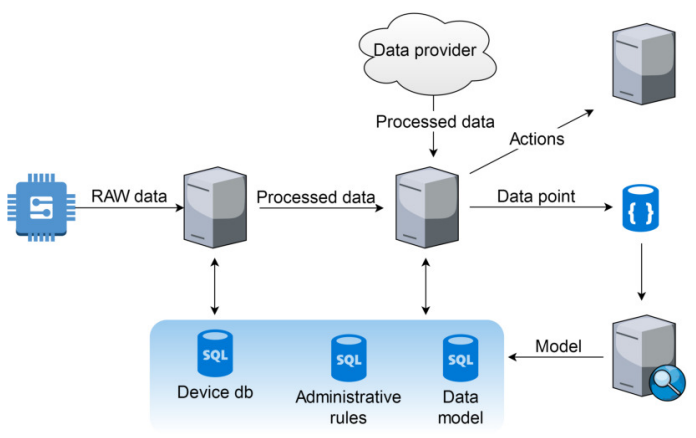

Fig. 7. See Your Box Architecture

The central processing node is based on a fully scalable infrastructure based on virtual machines that are responsible of fulfilling specific tasks. A lot of research and effort was invested in creating an efficient self load balancing system that could use the full potential of the available hardware. This allows the system to take advantage of instant and dynamic vertical scaling driven by the actual load of the system. The resulting architecture is summarized in figure 7 . The cloud platform is subdivided in three main component:

- Gateway, accepts incoming requests from devices, authenticates and decrypts data, forwards data within the system and delivers messages to devices.

- Rules Engine, applies business logic to the incoming data according to the risk sub model 
- Databases, the system uses a multiple DBMS approach to leverage the strengths of relational and non relational engines [4]

Details regarding the internal architecture of the system are beyond the scope of this article, however for completeness we will briefly consider in detail the element of task scheduling. The nature of communication channel used to transfer data from the smart sensing device to the cloud infrastructure is characterized by a very short timeout. The reference specification is that all incoming requests will timeout within a second.

The risk model that is used on the centralized node is structured as a set of rules that have a specific priority rank. All business logic is applied by executing these rules. Examples are data decryption, time synchronization or a change from a risk sub-model to another one altogether. Some of these rules are low latency (e.g. conversion of raw sensor data to a float value) while others are high latency due to complexity or interaction with external providers (e.g. compare sampled temperature with weather forecast service). Some rules have effects on the risk sub-model (e.g. a geofencing rule registers the arrival of a painting to the destination museum) while others don't (e.g. decrypt incoming data).

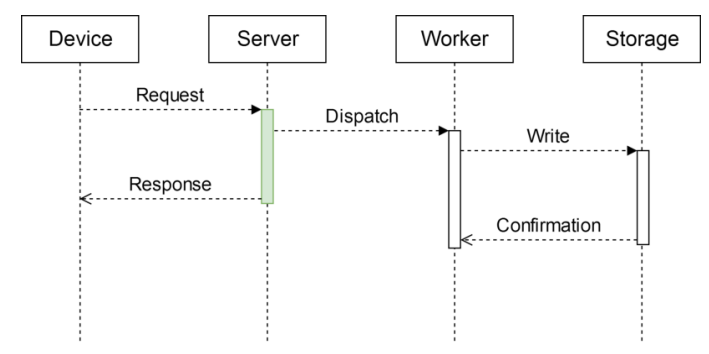

Fig. 8. A low priority high latency task is processed asynchronously

High priority tasks that are used to determine changes into the risk sub-model are placed into a synchronous queue so that effects of processing can be returned immediately to the smart sensing device. Low priority tasks are placed into an asynchronous queue so that we can close the communication channel as quickly as possible. Keeping the channel open for the minimum time necessary has a strong impact both on battery life for the smart sensing devices since all RF circuitry is active and on the performance of the cloud infrastructure since the gateway needs to be able to process massive quantities of requests per second.

\section{Web based risk monitoring dashboard}

The last component of our real time risk profile monitoring platform is the dashboard. It allows stakeholders involved in the inter-modal logistics process to have a real time over view of what is happening to the transported or stored painting. The dashboard was developed using AngularJS, a Javascript framework for single page applications and the layout of content was managed with the HTML/CSS framework Bootstrap. When using the dashboard users can:

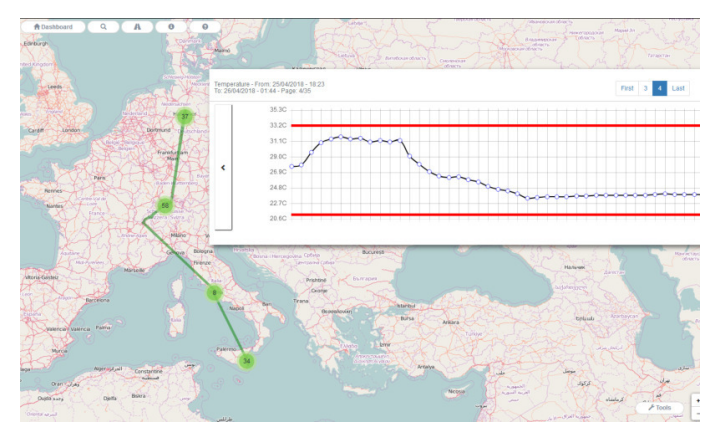

Fig. 9. Web based real time monitoring dashboard

- View the position of the painting over a map

- View time-series charts of all recorded environmental parameters

- Export data as CSV or PDF

- View the list of alerts generated during a monitoring activity

The dashboard was developed as a responsive web application that can be accessed also from mobile devices. Even though the true potential of the system lays in the M2M REST APIs that offer full integration with external sensors, the availability of a full fledged dashboard is essential in an inter-modal logistics process since multiple stakeholders might be part of different companies and a process of integration with each one would be slow.

\section{A Case Study}

The system presented in the previous chapter has been used to monitor over 200 real fine art international logistics transports and over 10 full inter-modal long term monitoring projects of temporary exhibitions that involved the coverage of both transport and exhibition. The logistics services were provided by a leading and specialized company for fine art transport while the exhibitions took place in museums amongst which Guggenheim and Vatican Museums. The duration of the long term monitoring projects, from nail-to-nail, ranged between 3 and 6 months. During these the system was able to swap into different configurations by accurately detecting the context of where the painting was placed. These swaps allowed the system to not only optimize power consumption, allowing six months of continuous real time monitoring while using a standard battery for mobile phones, but most importantly detect potential threats that were handled before they could actually damage the paintings.

As an example in figure 10 an inter-modal fine art logistics process over land and sea shows both the near miss and the trespassing of the critical temperature thresholds that the painting can withstand. Knowing the context the system was able to predict the risk for potential damage preventively during transport, allowing the logistics company to intervene in time and act upon the temperature control unit. In the second instance the system was able to ignore the potential threat since the combination of light levels, localization and movement 
were sufficient to detect the detachment of the sensor from the picture frame and therefore the end of the monitoring activity.

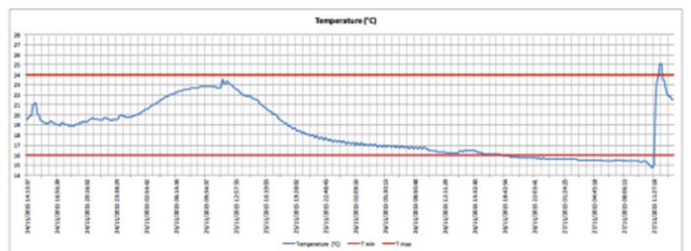

Fig. 10. Near miss of temperature thresholds

\section{CONCLuSions}

The paper discussed a systems, developed by See Your Box $^{\mathrm{TM}}$ Ltd., covering several problems dealing with the fineart logistics. The proposed architecture is an centralized cloud platform processing data collected from a distributed network of wireless sensors capable of edge computing that use extensively IoT and IIoT technologies.

Future developments of the system will allow for full integration for real time premium negotiation based on live risk levels.

\section{REFERENCES}

[1] "Research agenda for the application of ict to cultural heritage," Tech. Rep.

[2] C. Guccio, M. F. Martorana, I. Mazza, and I. Rizzo, Technology and Public Access to Cultural Heritage: The Italian Experience on ICT for Public Historical Archives. Cham: Springer International Publishing, 2016, pp. 55-75. [Online]. Available: https://doi.org/10. 1007/978-3-319-29544-2_4

[3] K. Yang, D. Forte, and M. M. Tehranipoor, "Protecting endpoint devices in iot supply chain," in Proceedings of the IEEE/ACM International Conference on Computer-Aided Design, ser. ICCAD '15. Piscataway, NJ, USA: IEEE Press, 2015, pp. 351-356. [Online]. Available: http://dl.acm.org/citation.cfm?id=2840819.2840869

[4] M. P. Loria, M. Toja, V. Carchiolo, and M. Malgeri, "An efficient realtime architecture for collecting iot data," in 2017 Federated Conference on Computer Science and Information Systems (FedCSIS), Sept 2017, pp. 1157-1166.

[5] V. Carchiolo, L. Compagno, M. Malgeri, N. Trapani, M. L. Previti, M. P. Loria, and M. Toja, "An efficient real-time monitoring to manage homebased oxygen therapy," in Trends and Advances in Information Systems and Technologies, A. Rocha, H. Adeli, L. P. Reis, and S. Costanzo, Eds. Cham: Springer International Publishing, 2018, pp. 741-749.
[6] A. Chianese, F. Piccialli, and G. Riccio, "The trust project: Improving the fruition of historical centres through smart objects," Procedia Computer Science, vol. 63, pp. 159 - 164, 2015, the 6th International Conference on Emerging Ubiquitous Systems and Pervasive Networks (EUSPN 2015)/ The 5th International Conference on Current and Future Trends of Information and Communication Technologies in Healthcare (ICTH-2015)/ Affiliated Workshops. [Online]. Available: http://www.sciencedirect.com/science/article/pii/S1877050915024552

[7] Z. He, B. Cui, W. Zhou, and S. Yokoi, "A proposal of interaction system between visitor and collection in museum hall by ibeacon," in 2015 10th International Conference on Computer Science Education (ICCSE), July 2015, pp. 427-430.

[8] M. Buzzi and C. Senette, RFID Sensors and Artifact Tracking. Cham: Springer International Publishing, 2017, pp. 435-451. [Online]. Available: https://doi.org/10.1007/978-3-319-50518-3_21

[9] S. Alletto, R. Cucchiara, G. D. Fiore, L. Mainetti, V. Mighali, L. Patrono, and G. Serra, "An indoor location-aware system for an iot-based smart museum," IEEE Internet of Things Journal, vol. 3, no. 2, pp. 244-253, April 2016.

[10] S. Cuomo, P. D. Michele, F. Piccialli, and A. K. Sangaiah, "Reproducing dynamics related to an internet of things framework: A numerical and statistical approach," Journal of Parallel and Distributed Computing, 2017. [Online]. Available: http://www.sciencedirect.com/science/article/ pii/S0743731517302095

[11] R. Mousheimish, Y. Taher, K. Zeitouni, and M. Dubus, "Smart preserving of cultural heritage with PACT-ART - enrichment, data mining, and complex event processing in the internet of cultural things," Multimedia Tools Appl., vol. 76, no. 24, pp. 26077-26101, 2017. [Online]. Available: https://doi.org/10.1007/s11042-017-4900-x

[12] R. Mousheimish, Y. Taher, and K. Zeitouni, "Toward the support of challenging service level agreements (slas) in manual and contextdependent activities," in 2016 IEEE 40th Annual Computer Software and Applications Conference (COMPSAC), vol. 2, June 2016, pp. 3843.

[13] R. Mousheimish, Y. Taher, K. Zeitouni, and M. Dubus, "Pact-art: Enrichment, data mining, and complex event processing in the internet of cultural things," in 2016 12th International Conference on Signal-Image Technology Internet-Based Systems (SITIS), Nov 2016, pp. 476-483.

[14] A. K. Hafsa Tahir and M. Junaid, "Internet of things (iot): An overview of applications and security issues regarding implementation," INTER NATIONAL JOURNAL OF MULTIDISCIPLINARY SCIENCES AND ENGINEERING, vol. 7, no. 1, 2016.

[15] G. S. Matharu, P. Upadhyay, and L. Chaudhary, "The internet of things: Challenges amp; security issues," in 2014 International Conference on Emerging Technologies (ICET), Dec 2014, pp. 54-59.

[16] "Furto gioielli: video riprende colpo, in tutto 20 secondi," 2018 (accessed 10/5/2018), http://www.ansa.it/veneto/ notizie/2018/01/03/furto-gioielli-a-palazzo-ducale-venezia_ 41f2d702-6270-4ba3-bae8-47d2b8a03dd2.html. 